\title{
Powerline Interference Suppression in High-Resolution ECG
}

\author{
A Bazhyna $^{1}$, II Christov $^{2}$, A Gotchev $^{1}$, IK Daskalov ${ }^{2}$, K Egiazarian $^{1}$ \\ ${ }^{1}$ Institute of Signal Processing, Tampere University of Technology, Tampere, Finland \\ ${ }^{2}$ Centre of Biomedical Engineering, Bulgarian Academy of Sciences, Sofia, Bulgaria
}

\begin{abstract}
The efficacy of four powerline interference suppression methods was tested for application in highresolution electrocardiogram (ECG). The goal was minimal distortion of the original signal micro-potential waveform, combined with maximum noise reduction. Simulated low amplitude His-bundle potentials were used for the evaluation. Several objective parameters were measured, such as mean square error and mean absolute error. The methods were applied for His-bundle potentials recovery from real surface ECG signals. Synchronous intracardiac signals with well expressed His potential were recorded and used for reference.

Modified time-domain subtraction and regression subtraction methods were found superior to notch filters and spectral interpolation.
\end{abstract}

\section{Introduction}

High resolution ECGs are most sensitive to parasite interference, as very low amplitude micro-potentials are to be extracted and analyzed. Most common disturbances arise from power-line interference, electromyogram (EMG) noise, motion artifacts and baseline wander (drift). The high-resolution ECG, being oriented toward detection and analysis of very low-amplitude potentials, should be combined very carefully with any noise suppression method in order to avoid introduction of signal distortions and miss-diagnostics. Not all the methods for noise suppression known and applied by far to 'standard' surface ECG acquisition and recording can be used in high-resolution ECG applications. Nowadays, there are still problems in EMG and powerline interference signals suppression in high-resolution ECG.

His-bundle potential amplitudes range from a few to about $20 \mu \mathrm{V}$ on the body surface. Therefore, the commonly used sampling rate is $1 \mathrm{kHz}$ or more with a resolution of about 50 to $100 \mathrm{nV} / \mathrm{bit}$.

Most often surface ECG micropotentials are obtained by serial signal averaging $[1,2]$, thus virtually avoiding the need of special procedures for powerline interference and EMG artifacts suppression. This is due mainly to the fact that no acceptable solution has been found for beatto-beat recording.

However, serial averaging has some known limitations, for example inapplicability for studying patients with certain types of irregular rhythms or with varying AV conduction [3]. Another drawback is the inability to extract micropotentials in transient rhythm disturbances which might be if clinical significance.

Beat-to-beat analyses require specific approaches for suppression of such dissimilar disturbances as powerline interference and EMG artifacts. The present study is mainly focused on powerline interference suppression.

Among the large variety of existing methods for mains interference suppression we selected four of the most well-known, and tested their performance towards minimal distortions of the original signal. The selected methods are: notch filters, spectral interpolation [4], timedomain subtraction method of proven quality in 'classic' surface ECG recording [5-8] and regression-subtraction [9]. The time-domain subtraction method was specially modified for His bundle recovery. The regression subtraction method, originally created for EMG, was adapted for mains suppression from ECG and changes related to better behavior with amplitude modulations of the interference during record were performed.

These methods were tested using ECGs corrupted by powerline interference and with added simulated Hisbundle micropotentials. The mean square error (MSE) and the mean absolute error (MAE) were used to estimate the performance. The same methods were applied to extract His-bundle signal form real surface ECGs with a synchronously recorded His-bundle intracardiac electrocardiogram.

\section{Methods}

\subsection{Time-domain subtraction}

The number of samples for one period of the powerline frequency $f_{0}$ and the sampling frequency $f_{S}$ is defined as $n=f_{S} / f_{0}$. A criterion is used to separate linearly evolving signal segments, further referred to as linear, from non-linear ones [5]: 


$$
\operatorname{abs}\left(D_{1}-D_{2}\right)<M,
$$

where $D_{1}$ and $D_{2}$ are two consecutive partial differences of the signal, at one and the same phase of the mains interference

$$
D_{1}=X_{i+n}-X_{i} ; \quad D_{2}=X_{i+1+n}-X_{i+1}
$$

and $M=100 \mu \mathrm{V}$ is a threshold.

In linear segments (the low-frequency content signal portions) a noise-free signal is obtained by applying a moving-average 'comb' filter:

$$
\begin{gathered}
Y_{(n+1) / 2}=\frac{\sum_{i=1}^{n} X_{i}}{n} \\
Y_{n / 2}=\frac{\sum_{i=1}^{n} X_{i}}{n}-\frac{X_{n+1}-X_{1}}{2 n},
\end{gathered}
$$

where $Y$ is the filtered signal, $X$ is the original one and $n$ is the number of samples in one period of the mains frequency.

Eq. (3) is to be used in case the selected sampling frequency has an odd number of samples per a $2 \pi$ interval of the interference, and Eq. (4) is for even number of samples, respectively.

The interference amplitude for a given phase is obtained by subtraction of the filtered sample from the original one. Next interference amplitudes are updated at each subsequent sample point (as interference amplitudes can vary) meeting the linearity criterion.

In non-linear segments (the QRS complex and some high-amplitude, steep-slope $T$ waves) noise free samples are obtained by subtraction of the calculated noise samples from the original signal.

This method is not directly applicable to body-surface His-ECG, as the low-amplitude and relatively lowfrequency His wave could not be distinguished and separated from linear segments. Thus the His wave was in fact suppressed or even removed from the signal. The EMG noise is usually of higher amplitude and much higher frequency content compared to the His-wave. Thus, a simple change of the threshold value $M$ did not result in an acceptable delineation of linear and nonlinear segments.

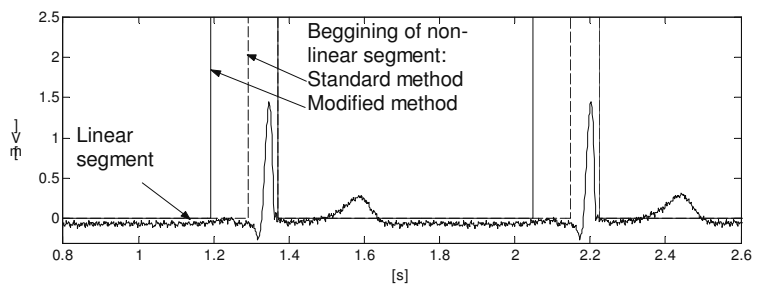

Figure 1. The beginning of a non-linear segment including the QRS complex was shifted $100 \mathrm{~ms}$ to the left, to include the zone where the His-bundle wave is expected to appear.

Therefore, the following modification was implemented. The beginning of the detected non-linear segment before a QRS complex was shifted $100 \mathrm{~ms}$ to the left, thus defining the His-wave region as non-linear segment by default (Figure 1).

\subsection{Regression-subtraction}

Regression-subtraction or time-correlated powerline interference subtraction [9] is a method based on the assumption that the interference is a sinusoidal signal of constant amplitude and phase during the interval of interest. Similarly to the method described in Subsection 2.1 , this method also relies to a segment where no informative signal activity is present. Having such a 'silent epoch' [9] or 'linear' segment [7], one can use it to estimate the amplitude and phase of the powerline contamination and to subtract it from subsequent samples in non-linear segments

We consider the T-P segments as intervals with no electrocardiogram activity and hence most appropriate for obtaining the powerline sinusoid. After a QRS detection the T-P segment is identified as beginning at $0.4 \sqrt{\mathrm{RR}} \mathrm{s}$ ( $R R$ is the averaged interval between $2 \mathrm{R}$ peaks) after the previous $\mathrm{R}$ peak and the T-P end is defined at $200 \mathrm{~ms}$ before the current $\mathrm{R}$ peak. To remove possible low frequency components in the defined T-P segment, high pass filtering is applied with cut-off frequency at $10 \mathrm{~Hz}$.

The powerline sinusoid can be represented as a superposition of two quadrature sinusoids having the same frequency:

$$
X_{k}^{(1)}=\sin \left(2 \pi f_{0} k / f_{s}\right) ; \quad X_{k}^{(2)}=\cos \left(2 \pi f_{0} k / f_{s}\right)
$$

The T-P segment denoted by $Y_{k}$ is considered as a sum of a periodical component and a zero mean random signal as follows:

$$
Y_{k}=a X_{k}^{(1)}+b X_{k}^{(2)}+e_{k} ;
$$

The problem of finding the coefficients $a$ and $b$ can be considered as a problem of projecting the given signal $Y_{k}$ onto the space of periodical signals with fixed frequency. For this space, the orthogonal signals $X^{(1)}$ and $X^{(2)}$ are the basis functions.

In the matrix form Eq. (6) is expressed as:

$$
\mathbf{Y}=\mathbf{X R}+\mathbf{e}
$$

where $\mathbf{Y}=[Y(1), Y(2), \ldots, Y(N)]^{\mathrm{T}}$ is the signal vector, $\mathbf{X}$ is $N \times 2$ matrix of orthogonal basis vectors $\mathbf{X}^{(1)}$ and $\mathbf{X}^{(2)}$, and $\mathbf{R}=[a, b]^{\mathrm{T}}$ is the vector of projection coefficients. $N$ is the length of the current segment.

The least-squares solution of this over-determined system of normal equations is well known from the matrix algebra [10] and is given by:

$$
\mathbf{R}=\left(\mathbf{X}^{\mathrm{T}} \mathbf{X}\right)^{-1} \mathbf{X}^{\mathrm{T}} \mathbf{Y}
$$

Eq. (8) is interpreted as follows: the signal $Y_{k}$ is multiplied by the basis sine and cosine waves for the current segment, that is $\mathbf{X}^{\mathrm{T}} \mathbf{Y}$. This operation requires $N \times 2$ multiplications and additions. The $2 \times 2$ matrix $\left(\mathbf{X}^{\mathrm{T}} \mathbf{X}\right)^{-1}$, grace to the orthogonal basis vectors, is just a 
normalization matrix in the form

$$
\left(\mathbf{X}^{\mathrm{T}} \mathbf{X}\right)^{-1}=\left[\begin{array}{cc}
2 / N & 0 \\
0 & 2 / N
\end{array}\right]
$$

The powerline sinusoid is assumed to have constant amplitude and phase for the whole heart beat, estimated as:

$$
p_{k}=a \sin \left(2 \pi f_{0} k / f_{s}\right)+b \cos \left(2 \pi f_{0} k / f_{s}\right)
$$

and is subtracted from it

$$
E C G_{k}=E C G_{k}-p_{k}
$$

The procedure is repeated for the next heart beat with new $a$ and $b$ regression coefficients to estimate the current sine amplitude and phase for subsequent subtraction. The same procedure can also be performed for harmonics of the mains frequency, i.e. for $f_{0}=150$, $250, \ldots \mathrm{Hz}$.

In general, a longer the T-P interval yields better coefficient estimation. However, thus the method is less sensitive to powerline interference amplitude changes. Also, longer interval requires more computations.

\section{Experiments and results}

Our experiments were performed on 16 timeconsecutive records (16.3 s duration each) of a patient from the high-resolution ECG database. Three leads were simultaneously recorded (two body-surface and one intracardiac) with $1000 \mathrm{~Hz}$ sampling rate and $100 \mathrm{nV} / \mathrm{bit}$ resolution. The surface ECGs were acquired from two bipolar chest leads, roughly similar to Frank $\mathrm{X}$ and $\mathrm{Y}$ frontal plane leads.
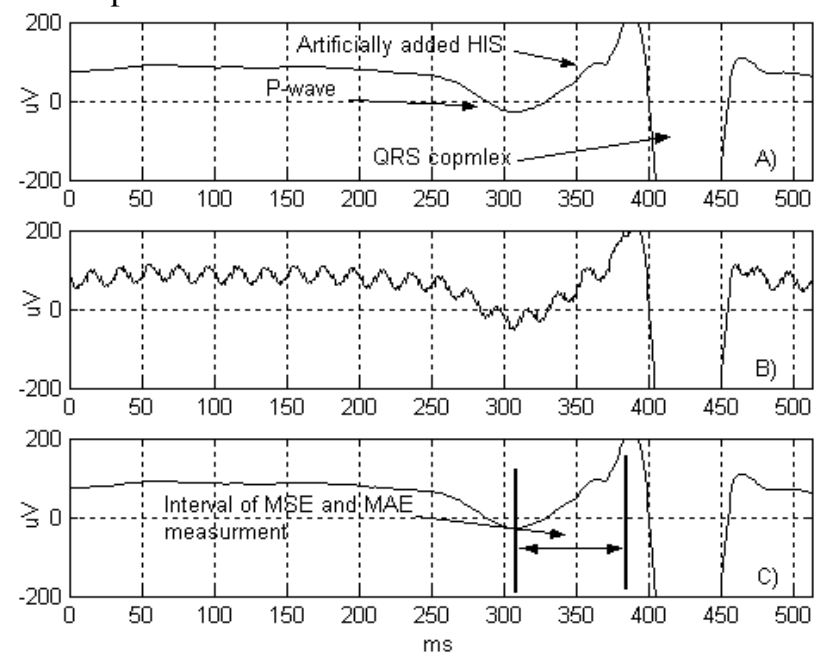

Figure 2. (A) Artificial His-bundle added in the P-Q segment of each heart ECG complex; (B) $50 \mathrm{~Hz}$ interference mixed with the entire record; (C) Processed signal.

Moving averaging filter was applied to practically eliminate powerline interference and to suppress EMG noise. His micropotentials were also eliminated, and then artificially added to the P-Q segment of every beat (Figure 2A). Powerline interference with $50 \mathrm{~Hz}$ frequency and its harmonics was generated and added. For each heartbeat every harmonic had random amplitude and initial phase. The powerline interference was added (Figure 2B) and then suppressed by each of the methods (Figure 2C).

The performance of algorithms was measured by mean-square error (MSE) and mean absolute error (MAE) calculated for the P-Q interval, as a difference between the restored (Figure 2C) and the original signal (Figure 2A).

The results for an initial signal-to-noise ratio (SNR) of $23 \mathrm{~dB}$ are presented in Table 1. For SNR of $14 \mathrm{~dB}$, MSE increases approximately by a factor of 1.5 for all methods except the subtraction ones, where the factor was about 1.1 .

It should be noted that artificial His bundle potential becomes visually detectable if MAE is smaller then 70$80 \mu V$ or equivalently if MSE is less then $3000-4000 \mu V^{2}$ on measured interval.

A series of experiments was made using the regression-subtraction method in order to determine how the estimation interval length (a part of T-P segment) affected the performance. The results shown in the Table 1 are for a length of $400 \mathrm{~ms}$. Decreasing the length to 300 ms increased twice the MSE and MAE. For shorter lengths (less then $200 \mathrm{~ms}$ ) the method's performance deteriorated substantially and became comparable to that of the notch filter.

Table 1. Experimental results for different mains interference suppression methods. MSE and MAE are averaged results for 120 heartbeats. The initial signal- tonoise ratio (SNR) is $23 \mathrm{~dB}$

\begin{tabular}{lll}
\hline Method & $\mathrm{MSE}, \mu V^{2}$ & $\mathrm{MAE}, \mu V$ \\
\hline Notch filter (Q-factor $=25)$ & 23483 & 126.4 \\
Notch filter (Q-factor = 100) & 1056 & 27.83 \\
Spectrum interpolation & 480.3 & 15.42 \\
Time-domain subtraction & 71.88 & 7.578 \\
Regression- subtraction & 33.46 & 4.221 \\
\hline
\end{tabular}

These methods were applied to real high-resolution ECG signals, corrupted by powerline interference and EMG noise

One of these signals is shown in Figure 3A. The result after processing by the modified regression-subtraction methods is shown on Figure 3B. The simultaneously recorded intracardiac signal, used to show the correct location of the surface signal His wave, is shown in Figure 3C.

Although the EMG noise in Figure 3B was not suppressed, the wave at about the $350^{\text {th }} \mathrm{ms}$ can be identified as a surface-detected His wave.

\section{Discussion}

The distortions introduced by notch filters makes them unacceptable for powerline interference removal from high-resolution ECG. Even with the high Q-factor, in 
presence of EMG noise, the result is below the visual detection threshold.
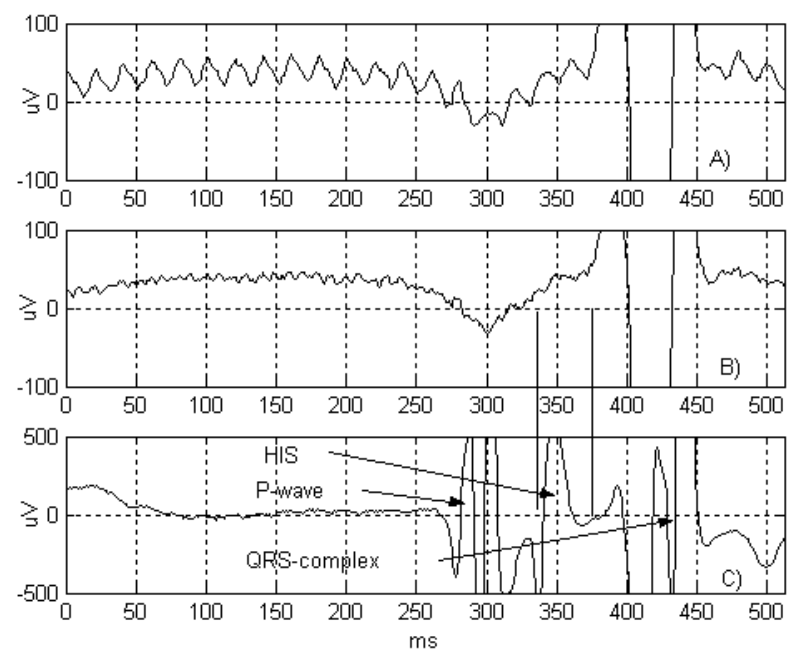

Figure 3. Powerline mains interference suppression performance of a real surface ECG signal. (A) Original signal with relatively low about $40 \mu \mathrm{Vp}$-p interference amplitude. (B) Processed signal using the regressionsubtraction method. C) synchronously recorded intracardiac signal.

The spectrum interpolation method introduces significantly smaller distortions to the original signal shape. However these distortions are still high due to the fact that the method did not properly deal with powerline frequency phase distortions.

The time-domain and regression-subtraction methods are similar in concept and use. They both rely on a lowfrequency content segment in the ECG, called either "linear" or "silent". From such a segment the powerline interference is extracted and subtracted from the relatively high-frequency ('non-linear') ECG segments.

The two methods principally differ in estimation of the sinusoid. In time-domain subtraction it is done by moving averaging in just $2 \pi$ interval. It is continually updated in the next $2 \pi$ for a 'linear' epoch. Errors are involved if there is not only mains interference, but also EMG noise in the 'linear' segment. Then the averaging does not give a clear estimate. Otherwise, the time-domain subtraction totally removes powerline interference and its harmonics with acceptable level of signal shape distortions. It also manages well with amplitude-modulated noise. A drawback of the method is that it operates perfectly only when the sampling frequency is in constant phase relationship with the powerline frequency. The efficiency decreases when the ECG is corrupted with huge EMG noise. The method is very attractive from computational point of view.

The modified regression subtraction estimates the sinusoid interference in a longer interval (T-P segment). It provides the lowest signal distortion among the reviewed methods and deals well with EMG noise, but does poorly with amplitude changes of the interference within the current segment.

Both time-domain methods deal properly with amplitude and phase changes of powerline interference thus providing for more accurate suppression.

The best powerline interference removal method should be chosen having in view beat-to-beat detection of ECG micropotentials, with a reasonable compromise between performance and computational complexity. Both time-domain methods should be considered as good candidates.

\section{Acknowledgements}

The authors gratefully acknowledge the contribution of Prof. Emil Novakov, de l'Université Josepf Fourier de Grenoble, for developing the high resolution ECG recording instrumentation and for participating in the acquisition of a series of recordings.

\section{References}

[1] Escalona OJ, Mitchell RH, Balderson DE, Harron DWG Fast and reliable QRS alignment technique for highfrequency analyses of signal-averaged ECG. Med and Biol Eng and Comp 1993;31:S137-S146.

[2] Turner IN, Wang W, English MJ, Vincent R. Noise coherence in closely-spaced electrodes: the implications for spatial averaged ECG recordings. Journ of Med Eng and Techn 1995;19:158-161.

[3] Flowers NC, Shvartsman V, Kennelly BM, Sohi GS, Horan LG. Surface recording of His-Purkinje activity on an every-beat basis without digital averaging. Circulation, 1981; 63:948-952.

[4] Mewett DT, Nazeran H, Reynolds KJ. Removing power line noise from recorded EMG. Eng in Med and Biol Soc Proc of 23rd Ann Int Conf IEEE 2000;3:2190-2193.

[5] Levkov C, Michov G, Ivanov R. Daskalov IK. Subtraction of $50 \mathrm{~Hz}$ interference from the ECG. Med Biol Eng Comput 1984;22:371-373.

[6] Christov II, Dotsinsky IA. New approach to the digital elimination of $50 \mathrm{~Hz}$ interference from the ECG. Med Biol. Eng Comput 1988;26: 431-434,

[7] Christov I. Dynamic powerline interference subtraction from biosignals. Jour of Med Eng and Tech. 2000;24:169172.

[8] Dotsinsky IA, Daskalov IK. Accuracy of the $50 \mathrm{~Hz}$ interference subtraction from the electrocardiogram. Med Biol Eng Comput 1996;34:489-494.

[9] Baratta RV, Solomonov M, Zhou BH, Zhu M. Methods to reduce the variability of EMG power spectrum estimates. Jour of EMG and Kinesiology 1998;8:279-285.

[10] Coleman TF, Van Loan CF. Handbook for Matrix Computations (Frontiers in Applied Mathematics, No 4), In: Ed SIAM, 2nd Edition, 1988.

Address for correspondence.

Andriy Bazhyna

Institute of Signal Processing, Tampere University of Technology, P.O.Box 553, FIN-33101 Tampere, FINLAND bazhyna@cs.tut.fi 